\title{
Bi-conformal symmetry and static Green functions in the Schwarzschild-Tangherlini spacetimes
}

\author{
Valeri P. Frolov and Andrei Zelnikov \\ Theoretical Physics Institute, Department of Physics, \\ University of Alberta, Edmonton, AB, T6G 2E1 Canada \\ E-mail: vfrolov@ualberta.ca, zelnikov@ualberta.ca
}

ABSTRACT: We study a static massless minimally coupled scalar field created by a source in a static D-dimensional spacetime. We demonstrate that the corresponding equation for this field is invariant under a special transformation of the background metric. This transformation consists of the static conformal transformation of the spatial part of the metric accompanied by a properly chosen transformation of the red-shift factor. Both transformations are determined by one function $\Omega$ of the spatial coordinates. We show that in a case of higher dimensional spherically symmetric black holes one can find such a bi-conformal transformation that the symmetry of the D-dimensional metric is enhanced after its application. Namely, the metric becomes a direct sum of the metric on a unit sphere and the metric of 2D anti-de Sitter space. The method of the heat kernels is used to find the Green function in this new space, which allows one, after dimensional reduction, to obtain a static Green function in the original space of the static black hole. The general useful representation of static Green functions is obtained in the Schwarzschild-Tangherlini spacetimes of arbitrary dimension. The exact explicit expressions for the static Green functions are obtained in such metrics for $D<6$. It is shown that in the four dimensional case the corresponding Green function coincides with the Copson solution.

Keywords: Conformal and W Symmetry, Field Theories in Higher Dimensions, Black Holes

ARXIV EPRINT: 1412.6719 


\section{Contents}

1 Introduction 1

2 Bi-conformal symmetry 3

2.1 Static Green function 3

2.2 Bi-conformal transformation of higher-dimensional static spherically symmetric spacetimes and symmetry enhancement 4

3 Scalar charges near static vacuum black hole 5

3.1 Schwarzschild-Tangherlini metric 5

$\begin{array}{lll}3.2 & \text { Boundary conditions } & 6\end{array}$

4 Scalar charges in the Bertotti-Robinson spacetime $\quad 8$

5 Green functions and heat kernels $\quad 9$

5.1 General relations 9

5.2 Heat kernel on $H^{2} \quad 9$

$\begin{array}{ll}5.3 \text { Heat kernel on } S^{n+1} & 10\end{array}$

6 Results 10

6.1 Odd $D \quad 11$

6.2 Example. $D=5 \quad 12$

$\begin{array}{lll}6.3 & \text { Even } D & 13\end{array}$

6.4 Example. $D=4 \quad 14$

6.5 Example. $D=6 \quad 15$

$\begin{array}{lll}7 & \text { Discussion } & 15\end{array}$

$\begin{array}{ll}\text { A Useful properties of the elliptic functions } & 16\end{array}$

\section{Introduction}

The problem of finding the field, the self-energy, and the self-force of charged particles near black holes has been attracting attention for more than 40 years [1-4], though the exact solution for the field of a point electric charge in the Schwarzschild geometry was first obtained much earlier [5]. Recent interest in the problem of the self-force is stimulated by a study of the back-reaction of the field on a particle moving near a black hole in connection with the gravitational wave emission by such particles. At the same time, even if a charged particle is at rest, the study of the self-force might be quite interesting in connection with different gedanken experiments with black holes. More recently, several publications discussed higher-dimensional aspects of this problem. This study was stimulated by general interest to spacetimes and brane models with large extra dimensions. 
In this paper we study the following problem: suppose a static charged particle, supported by some external force, is at rest close to a static black hole. We would like to know the field created by this charge. For simplicity we assume that a particle has a scalar charge which is the origin of a (minimally coupled) massless scalar field. Such a problem reduces to finding solutions of a special type of elliptic equation satisfying the regularity condition at the horizon and decreasing at infinity. A standard method is to expand a solution into modes obtained by proper separation of variables. However, using the corresponding series representation of the solution, for example, for the calculation of the self force, requires a lot of work which includes mode-by-mode subtraction of the divergences and summation of the obtained series (see e.g. [6]). In some special cases it is possible to use analytical approximations. For example, when a particle is close to the horizon one can find its field in a local domain in its vicinity by using the Rindler approximation (see, e.g., [7]). However in such an approximation the size of the domain must be much smaller than the gravitational radius and this approximation does not allows one to get information about a global structure of the field and its dependence on the topology of the horizon.

Fortunately, there are known interesting examples when the problem can be solved exactly and analytically. The best known example is a case of an electric charge near four-dimensional Schwarzschild black hole. This exact solution was obtained by Copson [5] and corrected by Linet [8] who demonstrated that an additional properly chosen zeromode solution must be added to the Copson expression in order to guarantee that the black hole is kept uncharged. The generalization of this solution to the case of a scalar charge was found in [9]. Further generalizations to the Reissner-Nordström and Kerr black holes can be found in $[3,4,10]$. Exact higher-dimensional solutions for a field of a static point charge near extremely charged black holes (or a set of such black holes) described by Majumdar-Papapetrou metrics were found in [11].

In this paper we propose a method which allows one to obtain solutions for the field of a charge in a variety of new interesting higher-dimensional black hole metrics. We shall describe this method in detail in the next section, but it might be useful to explain it without entering into details just now. We restrict here ourselves by a simplest case of a static point charge of a massless scalar field $\Phi$ in a D-dimensional static spacetime. Let us write the metric in the form $(a, b=1, \ldots, D-1)$

$$
d s^{2}=-\alpha^{2} d t^{2}+g_{a b} d x^{a} d x^{b}, \quad \partial_{t} \alpha=0, \quad \partial_{t} g_{a b}=0 .
$$

We consider a static scalar field $\Phi$ created by a static source $J$ in such a space. Let us write schematically the corresponding equation in the form

$$
\hat{F} \Phi=-4 \pi J,
$$

where $\hat{F}=\hat{F}_{[g, \alpha]}$ is a corresponding second order operator with coefficients depending on the $(D-1)$-dimensional metric $g_{a b}$ and the red-shift factor $\alpha$. We shall show that the form of this equation remains the same under rescaling

$$
g_{a b}=\Omega^{2} \bar{g}_{a b}, \quad \alpha=\Omega^{k_{\alpha}} \bar{\alpha}, \quad J=\Omega^{k_{J}} \bar{J},
$$

where $\Omega$ is a function of spatial coordinates $x^{a}$ and $k_{\alpha}$ and $k_{J}$ are properly chosen constants. 
Such a transformation is a special case of so-called bi-conformal transformations [12, 13]. In our case this is a usual conformal transformation of the $(D-1)$-dimensional metric $g_{a b}$ accompanied by a properly chosen rescaling of the red-shift factor $\alpha$. One can naturally interpret $\alpha$ as a $(D-1)$-dimensional dilaton field. Let us notice that the transformation (1.3) 'maps' the $D$-dimensional metric to another one, which in a general case is not a solution of the Einstein equations. However in special cases within this family of metrics, parametrized by a function $\Omega$, there may exist a metric which possesses an enhanced symmetry. This symmetry may make it possible to obtain the exact Green function in this new $D$-dimensional spacetime and to solve the problem (1.2). In this paper we demonstrate how this method works for a static Green function in the SchwarzschildTangherlini black-hole spacetimes and discuss its possible generalizations.

\section{Bi-conformal symmetry}

\subsection{Static Green function}

The action for the minimal scalar field $\Phi(X)$ is

$$
I=-\frac{1}{8 \pi} \int d X \sqrt{-g^{\mathrm{D}}} \Phi^{; \mu} \Phi_{; \mu}+\int d X \sqrt{-g^{\mathrm{D}}} J \Phi .
$$

Here $J(X)$ is a scalar charge density and $\sqrt{-g^{\mathrm{D}}}$ is the square root of the determinant of the $D$-dimensional metric $g_{\mu \nu}^{\mathrm{D}}$. The scalar field obeys the equation

$$
\square \Phi=-4 \pi J,
$$

where $\square$ is the standard covariant $D$-dimensional D'Alembertian. The solution of this equation can be written in terms of the retarded Green function $\mathbb{G}_{\mathrm{Ret}}\left(X, X^{\prime}\right)$

$$
\Phi(X)=4 \pi \int d X^{\prime} \sqrt{-g^{\mathrm{D}}\left(X^{\prime}\right)} \mathbb{G}_{\mathrm{Ret}}\left(X, X^{\prime}\right) J\left(X^{\prime}\right) .
$$

The retarded Green function satisfies the equation

$$
\square \mathbb{G}_{\mathrm{Ret}}\left(X, X^{\prime}\right)=-\delta\left(X, X^{\prime}\right)=-\frac{\delta\left(X-X^{\prime}\right)}{\sqrt{-g^{\mathrm{D}}}} .
$$

Let us assume that the spacetime is static with the metric (1.1), so that $X=\left(t, x^{a}\right)$ and

$$
\delta\left(X, X^{\prime}\right)=-\frac{\delta\left(t-t^{\prime}\right) \delta\left(x-x^{\prime}\right)}{\alpha\left(x^{\prime}\right) \sqrt{g\left(x^{\prime}\right)}} .
$$

The equation (2.2) for a static field $\Phi$ created by a static source $J$ takes the form (1.2) with

$$
\hat{F}=\frac{1}{\alpha \sqrt{g}} \partial_{a}\left(\alpha \sqrt{g} g^{a b} \partial_{b}\right)
$$

A solution of this equation can be written in the form

$$
\Phi(x)=4 \pi \int d x^{\prime} \alpha\left(x^{\prime}\right) \sqrt{g\left(x^{\prime}\right)} G\left(x, x^{\prime}\right) J\left(x^{\prime}\right)
$$


where $G\left(x, x^{\prime}\right)$ is a static Green function. The latter is a solution of the equation

$$
\hat{F} G\left(x, x^{\prime}\right)=-\frac{\delta\left(x-x^{\prime}\right)}{\alpha \sqrt{g}} .
$$

The current of a static point charge $q$ located at the point $y$ reads

$$
J(x)=q \frac{\delta(x-y)}{\sqrt{g}} .
$$

The scalar field at the point $x$ created by this charge is

$$
\Phi(x)=4 \pi q \alpha(y) G(x, y) .
$$

The $D$-dimensional Green function $\mathbb{G}_{\text {Ret }}\left(X, X^{\prime}\right)$ in a static spacetime depends on $t-t^{\prime}$ and a static Green function can be written as the time integral

$$
G\left(x, x^{\prime}\right)=\int_{-\infty}^{\infty} d t \mathbb{G}_{\mathrm{Ret}}\left(t, x ; 0, x^{\prime}\right) .
$$

\subsection{Bi-conformal transformation of higher-dimensional static spherically sym- metric spacetimes and symmetry enhancement}

Now let us consider a bi-conformal transformation (1.3) of the static metric (1.1). It is easy to check that the equation (1.2) preserves its form under this transformation provided

$$
k_{\alpha}=-(D-3), \quad k_{J}=2 .
$$

This bi-conformal invariance of the static field equation was used earlier for calculations of a self-energy and a self-force of charges in various gravitational backgrounds [7, 11, 1417]. This symmetry enables one to relate static Green functions for the fields in different spacetimes, for example, in the geometry of a set of extremely charged black holes (the Majumdar-Papapetrou spacetime) and in the flat Minkowski spacetime.

We focus now on a special class of metrics, namely static spherically symmetric metrics of the form $(n=D-3)$

$$
d s^{2}=-f(r) d t^{2}+f^{-1}(r) d r^{2}+r^{2} d \omega_{n+1}^{2}, \quad f=1-\frac{2 M}{r^{n}}+\frac{Q^{2}}{r^{2 n}},
$$

where $d \omega_{n+1}^{2}$ is the line element on the $(n+1)$-dimensional unit sphere. This is a metric of a charged $D$-dimensional black hole.

Let us make the bi-conformal transformation of this metric by using the conformal function

$$
\Omega=r / a,
$$

where $a$ is an arbitrary constant of a dimension of length. The transformed metric takes the form of a direct sum of two metrics

$$
d \bar{s}^{2}=d h^{2}+a^{2} d \omega_{n+1}^{2}, \quad d h^{2}=-\left(\frac{r}{a}\right)^{2 n} f(r) d t^{2}+\frac{a^{2}}{r^{2} f(r)} d r^{2} .
$$


It is easy to check that Ricci scalar $\bar{R}$ for the $2 \mathrm{D}$ metric $d h^{2}$ is constant

$$
\bar{R}=-2 n^{2} / a^{2} .
$$

This means that this 2D space is homogeneous. In fact this is a 2D anti-de Sitter metric. The symmetry $R^{1} \times \mathrm{SO}(n+2)$ of original spacetime after the bi-conformal transformation is enhanced and becomes $\mathrm{SO}(1,2) \times \mathrm{SO}(n+2)$.

In this paper we shall demonstrate how to use this enhanced symmetry in order to obtain a static Green in the Schwarzschild-Tangherlini metric describing a neutral static higher-dimensional black hole. The method does work for a case of higher-dimensional charged black holes as well, but the required calculations are more complicated and we discuss this case in another paper.

\section{Scalar charges near static vacuum black hole}

\subsection{Schwarzschild-Tangherlini metric}

The Schwarzschild-Tangherlini metric is a special case of $(2.13)$ with $Q=0$. It can be written as follows

$$
\begin{aligned}
d s^{2} & =-f d t^{2}+f^{-1} d r^{2}+r^{2} d \omega_{n+1}^{2}, \\
f & =1-\left(\frac{r_{\mathrm{g}}}{r}\right)^{n}, \quad r_{\mathrm{g}}=(2 M)^{1 / n}, \quad n=D-3 .
\end{aligned}
$$

Here $r_{\mathrm{g}}$ is the gravitational radius. The line element on the $(n+1)$-dimensional unit sphere $d \omega_{n+1}^{2}$ is defined by the following recursive relations

$$
d \omega_{n+1}^{2}=d \theta_{n}^{2}+\sin ^{2} \theta_{n} d \omega_{n}^{2}, \quad d \omega_{0}^{2}=d \phi^{2} .
$$

We denoted $\theta_{0} \equiv \phi \in[0,2 \pi]$. All other coordinates $\theta_{i>0} \in[0, \pi]$.

The static Green function in the Schwarzschild-Tangherlini spacetime (3.1) satisfies (2.8) with $\alpha=\sqrt{f}$. It is the solution of the equation

$$
\triangle G\left(x, x^{\prime}\right)=-\frac{\delta\left(r-r^{\prime}\right) \delta^{n+1}\left(\omega, \omega^{\prime}\right)}{r^{n+1}}, \quad \triangle=\frac{1}{r^{n+1}}\left(\partial_{r} r^{n+1} f \partial_{r}\right)+\frac{1}{r^{2}} \triangle_{\omega}^{n+1} .
$$

Here $\triangle_{\omega}^{n+1}$ and $\delta\left(\omega, \omega^{\prime}\right)$ are the Laplace operator and a covariant delta-function on the unit $(n+1)$-dimensional sphere, respectively,

$$
\begin{aligned}
\triangle_{\omega}^{n+1} & =\partial_{\theta_{n}}^{2}+n \frac{\cos \theta_{n}}{\sin \theta_{n}} \partial_{\theta_{n}}+\frac{1}{\sin ^{2} \theta_{n}} \triangle_{\omega}^{n}, & \triangle_{\omega}^{1} & =\partial_{\phi}^{2}, \\
\delta^{n+1}\left(\omega, \omega^{\prime}\right) & =\frac{\delta\left(\theta_{n}-\theta_{n}^{\prime}\right)}{\sin ^{n} \theta_{n}} \delta^{n}\left(\omega, \omega^{\prime}\right), & \delta^{1}\left(\omega, \omega^{\prime}\right) & =\delta\left(\phi-\phi^{\prime}\right) .
\end{aligned}
$$

It is convenient to introduce a new radial variable $\rho$ related to the Schwarzschild radial coordinate $r$ as follows

$$
\frac{r}{r_{\mathrm{g}}}=\left(\frac{\rho+1}{2}\right)^{1 / n}, \quad \rho=2 \frac{r^{n}}{r_{\mathrm{g}}^{n}}-1 .
$$


The Tangherlini metric takes the form

$$
d s^{2}=-\frac{\rho-1}{\rho+1} d t^{2}+r_{g}^{2}\left(\frac{\rho+1}{2}\right)^{2 / n}\left[\frac{1}{n^{2}\left(\rho^{2}-1\right)} d \rho^{2}+d \omega_{n+1}^{2}\right] .
$$

Using relations

$$
\frac{\delta\left(r-r^{\prime}\right)}{r^{n-1}}=\frac{2 n}{r_{\mathrm{g}}^{n}} \delta\left(\rho-\rho^{\prime}\right), \quad \partial_{r}=\frac{2 n}{r_{\mathrm{g}}}\left(\frac{\rho+1}{2}\right)^{(n-1) / n} \partial_{\rho},
$$

one can show that the equation (3.3) for the static Green function takes the form

$$
\left[n^{2}\left(\rho^{2}-1\right) \partial_{\rho}^{2}+2 n^{2} \rho \partial_{\rho}+\triangle_{\omega}^{n+1}\right] G\left(x, x^{\prime}\right)=-\frac{2 n}{r_{g}^{n}} \delta\left(\rho-\rho^{\prime}\right) \delta^{n+1}\left(\omega, \omega^{\prime}\right) .
$$

The scalar field $\Phi(x)$ of a point charge $q$ located at $y$ is given by (2.10).

\subsection{Boundary conditions}

The horizon, where $\rho=1$, is a singular point of the equation (3.8). In order to specify uniquely a solution of this equation one needs, besides a natural fall off condition at infinity a specially chosen boundary condition at $\rho=1$. This condition follows from the requirement that the field be regular at the horizon in regular coordinates that cover it. Let us discuss this point in more detail.

In a physical set up, our problem can be formulated as follows. Let a spherically symmetric black hole be formed by a collapse of a spherical object. The metric outside this object is always (before and after black hole formation) described by the SchwarzschildTangherlini solution. We assume that before and after the collapse there are no free waves of the scalar field. The form of the solution (2.11) reflects this. Let us consider now a static charge in the spacetime of the eternal black hole with the same parameters. The late time solution for the field $\Phi$ is the same in both cases (an eternal black hole and a black hole created by collapse). Thus, one can start with a solution $\Phi$ for a static source $J$ placed near the eternal black hole. By causality, this solution is defined only outside the past horizon $H^{-}$. Beyond this surface it can have an arbitrary continuation. However, in order to avoid a singularity on $H^{-}$, one needs to assume a special continuity property of $\Phi$. This problem and the how to deal with it was discussed in $[4,18,19]$. Because the source $J$ is static, there is no radiation and, hence, radiation reaction terms and the field $\Phi$ in the black hole exterior remain the same. The natural way is to consider a time-symmetric part [20] of $\mathbb{G}_{\text {Ret }}$. In particular, the value of the field on the horizons $H^{ \pm}$, which are functions of the angular variables, are the same and coincide with the value of the field on the bifurcation surface of the horizons. As the result, one can analytically continue the static field $\Phi$ to the whole spacetime of the eternal black hole. After a Wick rotation, the static field $\Phi$ is regular at the Euclidean horizon. This property can be used to obtain the proper boundary conditions for a static equation (3.8) at its singular point $\rho=1$.

Let us make the Wick rotation $t=i t_{\mathrm{E}}$ in the metric (3.6) and consider the vicinity of the Euclidean horizon. Let us write $\rho$ in the form

$$
\rho=1+\frac{n^{2}}{2 r_{g}^{2}} \ell^{2}+\ldots
$$


$\ell=0$ is a position of the horizon and $\ell$ is a proper length distance from it. Here (..) denotes terms of higher order in $\ell^{2}$. The Euclidean version of the metric (3.6) takes the form

$$
d s_{E}^{2} \approx \frac{n^{2}}{4 r_{g}^{2}} \ell^{2} d t_{\mathrm{E}}^{2}+d \ell^{2}+r_{g}^{2} d \omega_{n+1}^{2} .
$$

The regularity of the Euclidean horizon requires that the coordinate $t_{\mathrm{E}}$ be periodic with the period

$$
\beta=\frac{2 \pi}{\kappa}=\frac{4 \pi r_{\mathrm{g}}}{n},
$$

where $\kappa$ is the surface gravity,

$$
\kappa=\frac{n}{2 r_{\mathrm{g}}} .
$$

The operator $\hat{F}$ near the horizon is

$$
\hat{F} \approx\left(\partial_{\ell}^{2}+\frac{1}{\ell} \partial_{\ell}\right)+\frac{1}{r_{\mathrm{g}}^{2}} \triangle_{\omega}^{n+1} .
$$

Let us write a solution of the equation

$$
\hat{F} \Phi=0
$$

near the horizon in the form

$$
\Phi=f_{0}+f_{1} \ell+f_{2} \ell^{2}+\ldots,
$$

where $f_{i}$ are functions on a unit sphere. Then (3.14) implies

$$
f_{1}=0, \quad f_{2}=-\frac{1}{4 r_{\mathrm{g}}^{2}} \triangle_{\omega}^{n+1} f_{0} .
$$

This means that $\Phi$ is a regular function near the Euclidean horizon. This is a proper boundary condition for $\Phi$ near $\rho=1$.

For any solution of (3.8) one can always add a solution of a homogeneous equation with the same operator. This arbitrariness is related to the zero-modes contribution to the Green function. These extra zero-mode terms in the Green function have to be symmetric in $x$ and $x^{\prime}$ and regular outside the horizon. In our case of the (3.8) they are characterized by two arbitrary constants $C_{1}$ and $C_{2}$

$$
C_{1} \ln \left(\frac{\rho-1}{\rho+1}\right) \ln \left(\frac{\rho^{\prime}-1}{\rho^{\prime}+1}\right)+C_{2}\left[\ln \left(\frac{\rho-1}{\rho+1}\right)+\ln \left(\frac{\rho^{\prime}-1}{\rho^{\prime}+1}\right)\right] .
$$

Using these terms one can adjust the solution for the scalar field to make it regular on the horizon and satisfy proper boundary conditions at infinity.

Let us notice that if one makes a bi-conformal transformation with $\Omega$ regular on the horizon $\Omega=\Omega_{0}+\Omega_{2} \ell^{2}+\ldots$, then the surface gravity $\kappa$ changes as follows $\bar{\kappa}=\Omega_{0}^{n+1} \kappa$. 


\section{Scalar charges in the Bertotti-Robinson spacetime}

Let us make a bi-conformal transformation of the Tangherlini metric

$$
g_{a b}=\Omega^{2} \bar{g}_{a b}, \quad \alpha=\Omega^{-n} \bar{\alpha},
$$

by choosing the conformal factor (2.14) with $a=r_{\mathrm{g}}$

$$
\Omega=\frac{r}{r_{\mathrm{g}}}=\left(\frac{\rho+1}{2}\right)^{1 / n}
$$

Notice that at the horizon $\Omega=1$ so that this transformation does not modify the surface gravity (3.12). The Tangherlini metric (3.6) transforms to

$$
d \bar{s}^{2}=\left(\frac{r_{\mathrm{g}}}{n}\right)^{2}\left[-\kappa^{2}\left(\rho^{2}-1\right) d t^{2}+\left(\rho^{2}-1\right)^{-1} d \rho^{2}\right]+r_{\mathrm{g}}^{2} d \omega_{n+1}^{2} .
$$

Its Euclidean version is the metric on $H^{2} \times S^{n+1}$ and has the form

$$
d \bar{s}_{\mathrm{E}}^{2}=\left(\frac{r_{\mathrm{g}}}{n}\right)^{2}\left[\kappa^{2}\left(\rho^{2}-1\right) d t_{\mathrm{E}}^{2}+\left(\rho^{2}-1\right)^{-1} d \rho^{2}\right]+r_{\mathrm{g}}^{2} d \omega_{n+1}^{2} .
$$

Let us notice that the metric (4.3) is a special case of the Bertotti-Robinson metric

$$
d s^{2}=b^{2} d H^{2}+a^{2} d \omega_{n+1}^{2}
$$

with $b=r_{\mathrm{g}} / n$ and $a=r_{\mathrm{g}}$. Here

$$
d H^{2}=-\left(\rho^{2}-1\right) d \bar{\sigma}^{2}+\left(\rho^{2}-1\right)^{-1} d \rho^{2}
$$

is the metric of the two-dimensional AdS spacetime of a unit curvature radius. The coordinate $\bar{\sigma}$ is related to the Schwarzschild time as follows $\bar{\sigma}=\kappa t$.

After the Wick rotation $\bar{\sigma}=i \sigma$ this metric takes the form

$$
d H_{\mathrm{E}}^{2}=\left(\rho^{2}-1\right) d \sigma^{2}+\left(\rho^{2}-1\right)^{-1} d \rho^{2} .
$$

It is regular at the Euclidean horizon $\rho=1$ provided $\sigma$ is periodic with the period $2 \pi$. The scalar curvatures of the sphere $S^{n+1}$ of the radius $a$ and of the hyperboloid $H^{2}$ of the radius $b$ are

$$
R_{S^{n+1}}=\frac{n(n+1)}{a^{2}}, \quad R_{H^{2}}=-\frac{2}{b^{2}} .
$$

Denote by $\mathbb{G}$ the Euclidean Green function of the operator $\square_{E}$ defined on the Euclidean Bertotti-Robinson metric (4.4). It is the solution of the equation

$$
\bar{\square}_{\mathrm{E}} \mathbb{G}\left(X_{\mathrm{E}}, X_{\mathrm{E}}^{\prime}\right)=-\delta\left(X_{\mathrm{E}}, X_{\mathrm{E}}^{\prime}\right),
$$

decreasing at infinity and regular at the Euclidean horizon $\rho=1$. We define the static Green function $G^{\mathrm{BR}}\left(x, x^{\prime}\right)$ as

$$
G^{\mathrm{BR}}\left(x, x^{\prime}\right)=\int_{0}^{\beta} d t_{\mathrm{E}} \mathbb{G}\left(t_{\mathrm{E}}, x ; 0, x^{\prime}\right) .
$$


Then the static Green function of the minimally coupled massless scalar field on the Bertotti-Robinson spacetime can be calculated as the integral of the Euclidean Green function of the scalar field in the Bertotti-Robinson spacetime. It is regular at the horizon, decreases at infinity, and satisfies the equation

$$
\left[n^{2}\left(\rho^{2}-1\right) \partial_{\rho}^{2}+2 n^{2} \rho \partial_{\rho}+\triangle_{\omega}^{n+1}\right] G^{\mathrm{BR}}\left(x, x^{\prime}\right)=-\frac{2 n}{r_{\mathrm{g}}^{n}} \delta\left(\rho-\rho^{\prime}\right) \delta^{n+1}\left(\omega, \omega^{\prime}\right),
$$

which coincides identically with the equation (3.8) for the static Green function on the Schwarzschild-Tangherlini background. This means that

$$
G^{\mathrm{BR}}\left(x, x^{\prime}\right)=G\left(x, x^{\prime}\right),
$$

where $G\left(x, x^{\prime}\right)$ is the static Green function for the original Schwarzschild-Tangherlini spacetime.

\section{Green functions and heat kernels}

\subsection{General relations}

We demonstrated that the static Green function in the Schwarzschild-Tangherlini spacetime can be found as soon as the corresponding Green function for the Bertotti-Robinson geometry is known. For this purpose it is convenient to use the heat kernel technique. Namely, the Euclidean Green function for the operator $\square_{E}$ is

$$
\mathbb{G}\left(X_{\mathrm{E}}, X_{\mathrm{E}}^{\prime}\right)=\int_{0}^{\infty} d s K\left(s \mid X_{\mathrm{E}}, X_{\mathrm{E}}^{\prime}\right) .
$$

Here the heat kernel $K\left(s \mid X_{\mathrm{E}}, X_{\mathrm{E}}^{\prime}\right)$ is the solution of the problem

$$
\left(\partial_{s}-\square_{\mathrm{E}}\right) K\left(s \mid X_{\mathrm{E}}, X_{\mathrm{E}}^{\prime}\right)=0, \quad K\left(0 \mid X_{\mathrm{E}}, X_{\mathrm{E}}^{\prime}\right)=\delta\left(X_{\mathrm{E}}, X_{\mathrm{E}}^{\prime}\right),
$$

which satisfies the same boundary conditions with respect to its arguments $X_{\mathrm{E}}$ and $X_{\mathrm{E}}^{\prime}$ as the Green function in question.

The Bertotti-Robinson metric (4.7) is the direct sum of two geometries $H^{2}$ and $S^{n+1}$. This implies that the heat kernel $K$ is the product of the heat kernels $K_{H^{2}}$ and $K_{S^{n+1}}$ on the hyperboloid $H^{2}$ and on the sphere $S^{n+1}$, correspondingly. Both spaces are homogeneous and isotropic and the corresponding heat kernels are known explicitly [21].

\section{$5.2 \quad$ Heat kernel on $\boldsymbol{H}^{2}$}

The heat kernel on $H^{2}$ of the radius $b$

$$
d H_{\mathrm{E}}^{2}=b^{2}\left[\left(\rho^{2}-1\right) d \sigma^{2}+\left(\rho^{2}-1\right)^{-1} d \rho^{2}\right]
$$

reads $[21]$

$$
K_{H^{2}}(s \mid \chi)=\frac{\sqrt{2} b}{(4 \pi s)^{3 / 2}} e^{-s /\left(4 b^{2}\right)} \int_{\chi}^{\infty} d y \frac{y e^{-b^{2} y^{2} /(4 s)}}{(\cosh y-\cosh (\chi))^{1 / 2}} .
$$

Here $\chi$ is the geodesic distance between two points on the $H^{2}$ of the unit radius $b=1$. It can be found from the relation

$$
\cosh (\chi)=\rho \rho^{\prime}-\sqrt{\rho^{2}-1} \sqrt{\rho^{\prime 2}-1} \cos \left(\sigma-\sigma^{\prime}\right) .
$$




\subsection{Heat kernel on $S^{n+1}$}

The heat kernel on $S^{2}$ of the radius $a$ reads [21]

$$
K_{S^{2}}(s \mid \gamma)=\frac{\sqrt{2} a}{(4 \pi s)^{3 / 2}} e^{s /\left(4 a^{2}\right)} \sum_{k=-\infty}^{\infty}(-1)^{k} \int_{\gamma}^{\pi} d \phi \frac{(\phi+2 \pi k) e^{-a^{2}(\phi+2 \pi k)^{2} /(4 s)}}{(\cos \gamma-\cos \phi)^{1 / 2}}
$$

Another equivalent representation of this kernel is

$$
K_{S^{2}}(s \mid \gamma)=\frac{1}{4 \pi a^{2}} \sum_{l=0}^{\infty}(2 l+1) P_{l}(\cos \gamma) e^{-\frac{s l(l+1)}{a^{2}}} .
$$

Here $\gamma$ is the geodesic distance between two points on the unit $S^{2}(a=1)$

$$
\cos \gamma=\cos \left(\theta_{1}\right) \cos \left(\theta_{1}^{\prime}\right)+\sin \left(\theta_{1}\right) \sin \left(\theta_{1}^{\prime}\right) \cos \left(\phi-\phi^{\prime}\right) .
$$

The heat kernel on $S^{3}$ of the radius $a$ reads [21]

$$
K_{S^{3}}(s \mid \gamma)=\frac{1}{(4 \pi s)^{3 / 2}} e^{s / a^{2}} \sum_{k=-\infty}^{\infty} \frac{(\gamma+2 \pi k) e^{-a^{2}(\gamma+2 \pi k)^{2} /(4 s)}}{\sin \gamma}
$$

where $\gamma$ is the geodesic distance between two points on the unit $S^{3}(a=1)$

$$
\cos \gamma=\cos \left(\theta_{2}\right) \cos \left(\theta_{2}^{\prime}\right)+\sin \left(\theta_{2}\right) \sin \left(\theta_{2}^{\prime}\right)\left[\cos \left(\theta_{1}\right) \cos \left(\theta_{1}^{\prime}\right)+\sin \left(\theta_{1}\right) \sin \left(\theta_{1}^{\prime}\right) \cos \left(\phi-\phi^{\prime}\right)\right] .
$$

The heat kernels on $S^{n+1}$ can be derived from $K_{S^{2}}$ and $K_{S^{3}}$ using the relations (see [21] eq. (8.12)-eq. (8.13))

$$
\begin{gathered}
K_{S^{n+1}}(s \mid \gamma)=e^{\frac{\left(n^{2}-1\right) s}{4 a^{2}}}\left(\frac{1}{2 \pi a^{2}} \frac{\partial}{\partial \cos \gamma}\right)^{\frac{(n-1)}{2}} K_{S^{2}}(s \mid \gamma), \quad n \text { odd } \\
K_{S^{n+1}}(s \mid \gamma)=e^{\frac{\left(n^{2}-4\right) s}{4 a^{2}}}\left(\frac{1}{2 \pi a^{2}} \frac{\partial}{\partial \cos \gamma}\right)^{\frac{(n-2)}{2}} K_{S^{3}}(s \mid \gamma), \quad n \text { even } .
\end{gathered}
$$

\section{Results}

Combining the results of the previous section together one obtains

$$
K\left(s \mid X_{\mathrm{E}}, X_{\mathrm{E}}^{\prime}\right)=K(s \mid \chi, \gamma)=K_{H^{2}}(s \mid \chi) \times K_{S^{n+1}}(s \mid \gamma) .
$$

Because of the symmetry of the Euclidean Bertotti-Robinson space the heat kernel $K$ can be written as the function of only three variables: the proper time $s$ and geodesic distances $\chi$ and $\gamma$ on the unit hyperboloid and the unit sphere.

In what follows we use the relation $b=a / n$ and identify $a$ with $r_{\mathrm{g}}$. For brevity we put $a=r_{\mathrm{g}}$ only at the end of calculations. One should keep in mind that the cases of spacetimes of even and odd dimensions differ qualitatively, and should be studied separately. 


\section{$6.1 \quad$ Odd $D$}

For odd $D($ even $n)$

$$
\begin{aligned}
K(s \mid \chi, \gamma)=\frac{a}{n}\left(\frac{1}{2 \pi a^{2}} \frac{\partial}{\partial \cos \gamma}\right)^{(n-2) / 2} \frac{\sqrt{2}}{(4 \pi s)^{3}} \frac{1}{\sin \gamma} & \\
& \sum_{k=-\infty}^{\infty}(\gamma+2 \pi k) e^{-a^{2}(\gamma+2 \pi k)^{2} /(4 s)} \int_{\chi}^{\infty} d y \frac{y e^{-a^{2} y^{2} /\left(4 n^{2} s\right)}}{(\cosh y-\cosh \chi)^{1 / 2}} .
\end{aligned}
$$

Equivalently one can write

$$
\begin{aligned}
K(s \mid \chi, \gamma)=\frac{a}{n}\left(\frac{1}{2 \pi a^{2}} \frac{\partial}{\partial \cos \gamma}\right)^{n / 2} \frac{\sqrt{2}}{(4 \pi s)^{2}} & \\
& \sum_{k=-\infty}^{\infty} e^{-a^{2}(\gamma+2 \pi k)^{2} /(4 s)} \int_{\chi}^{\infty} d y \frac{y e^{-a^{2} y^{2} /\left(4 n^{2} s\right)}}{(\cosh y-\cosh \chi)^{1 / 2}}
\end{aligned}
$$

The Green function is an integral of the heat kernel over $s$

$$
\mathbb{G}(\chi, \gamma)=\int_{0}^{\infty} d s K(s \mid \chi, \gamma) \text {. }
$$

For separated points we can perform this integration first

$$
\begin{aligned}
\mathbb{G}(\chi, \gamma)=\frac{1}{a n} \frac{\sqrt{2}}{4 \pi^{2}} & \left(\frac{1}{2 \pi a^{2}} \frac{\partial}{\partial \cos \gamma}\right)^{n / 2} \times \\
& \times \sum_{k=-\infty}^{\infty} \int_{\chi}^{\infty} d y \frac{y}{(\cosh y-\cosh \chi)^{1 / 2}} \frac{1}{\left[\frac{y^{2}}{n^{2}}+(\gamma+2 \pi k)^{2}\right]} .
\end{aligned}
$$

Summation over $k$ gives

$$
\sum_{k=-\infty}^{\infty} \frac{1}{\left[\frac{y^{2}}{n^{2}}+(\gamma+2 \pi k)^{2}\right]}=\frac{n}{2 y} \frac{\sinh \left(\frac{y}{2 n}\right) \cosh \left(\frac{y}{2 n}\right)}{\cosh ^{2}\left(\frac{y}{2 n}\right)-\cos ^{2}\left(\frac{\gamma}{2}\right)}=\frac{n}{2 y} \frac{\sinh \left(\frac{y}{n}\right)}{\cosh \left(\frac{y}{n}\right)-\cos \gamma} .
$$

Therefore, the $D$-dimensional Euclidean Green function on the Bertotti-Robinson spacetime becomes

$$
\mathbb{G}(\chi, \gamma)=\frac{\sqrt{2}}{8 \pi^{2} a}\left(\frac{1}{2 \pi a^{2}} \frac{\partial}{\partial \cos \gamma}\right)^{n / 2} \int_{\chi}^{\infty} d y \frac{1}{(\cosh y-\cosh \chi)^{1 / 2}} \frac{\sinh (y / n)}{\cosh (y / n)-\cos \gamma},
$$

where $\chi$ is given by (5.5).

This is the Euclidean Green function of a massless minimally coupled scalar field on the background of the Euclidean $D$-dimensional (odd $D$ ) Bertotti-Robinson spacetime. This Green function can be used, e.g., for the computation of the vacuum mean value of the stress-energy tensor or $\left\langle\Phi^{2}\right\rangle$ on the higher-dimensional Bertotti-Robinson geometry. In four dimensions the Green function in closed form was found in [22] and was used for the calculation of the vacuum mean value of the stress-energy tensor in [23]. 
Using the properties (4.10),(4.12) we finally get the static Green function on the odddimensional Schwarzschild-Tangherlini spacetimes

$$
G\left(x, x^{\prime}\right)=\frac{\sqrt{2}}{4 \pi^{2} n}\left(\frac{1}{2 \pi a^{2}} \frac{\partial}{\partial \cos \gamma}\right)^{n / 2} \int_{0}^{2 \pi} d \sigma \int_{\chi}^{\infty} d y \frac{1}{(\cosh y-\cosh \chi)^{1 / 2}} \frac{\sinh (y / n)}{\cosh (y / n)-\cos \gamma},
$$

where $x=\left(\rho, \theta_{i}\right), a=r_{\mathrm{g}}$, and

$$
\cosh (\chi)=\rho \rho^{\prime}-\sqrt{\rho^{2}-1} \sqrt{\rho^{\prime 2}-1} \cos \sigma, \quad \rho=2 \frac{r^{n}}{r_{\mathrm{g}}^{n}}-1 .
$$

\subsection{Example. $D=5$}

In five dimensions $(n=2)$ we have

$$
\begin{aligned}
\mathbb{G}(\chi, \gamma) & =\frac{\sqrt{2}}{8 \pi^{2} a}\left(\frac{1}{2 \pi a^{2}} \frac{\partial}{\partial \cos \gamma}\right) \int_{\chi}^{\infty} d y \frac{1}{\sqrt{\cosh y-\cosh \chi}} \frac{\sinh (y / 2)}{\cosh (y / 2)-\cos \gamma} \\
& =\frac{1}{(2 \pi a)^{3}}\left(\frac{\partial}{\partial \cos \gamma}\right)\left\{\lambda\left[\arctan (\lambda \cos \gamma)+\frac{\pi}{2}\right]\right\}
\end{aligned}
$$

where

$$
\lambda=\left(\cosh ^{2}\left(\frac{\chi}{2}\right)-\cos ^{2} \gamma\right)^{-1 / 2} .
$$

Performing the differentiation, we obtain a closed form for the Euclidean Green function in the Euclidean Bertotti-Robinson spacetime (4.4)

$$
\mathbb{G}(\chi, \gamma)=\frac{1}{(2 \pi a)^{3}}\left\{\lambda^{3} \cos \gamma\left[\arctan (\lambda \cos \gamma)+\frac{\pi}{2}\right]-\lambda^{2}\right\}
$$

The static Tangherlini Green function can be written as follows

$$
G\left(x, x^{\prime}\right)=a \int_{0}^{2 \pi} d \sigma \mathbb{G}(\chi, \gamma), \quad a=r_{\mathrm{g}} .
$$

The result of the integration over the Euclidean time $\sigma$ can be expressed in terms of elliptic functions. Using the properties of the elliptic functions presented in the appendix A we obtain the static Green function in the five-dimensional Bertotti-Robinson spacetime

$$
G\left(x, x^{\prime}\right)=\frac{1}{4 \pi^{2} r_{\mathrm{g}}^{2}} \frac{1}{\left(\rho^{2}-1\right)^{1 / 4}\left(\rho^{2}-1\right)^{1 / 4}}\left(\frac{\partial}{\partial \cos \gamma}\right) \varkappa[\mathbf{F}(\psi, \varkappa)+\mathbf{K}(\varkappa)]
$$

where

$$
\begin{aligned}
\sin \psi & =\cos \gamma \frac{\sqrt{2}}{\sqrt{\rho \rho^{\prime}-\sqrt{\rho^{2}-1} \sqrt{\rho^{\prime 2}-1}+1}}, \\
\varkappa & =\frac{\sqrt{2}\left(\rho^{2}-1\right)^{1 / 4}\left(\rho^{\prime 2}-1\right)^{1 / 4}}{\sqrt{\rho \rho^{\prime}+\sqrt{\rho^{2}-1} \sqrt{\rho^{\prime 2}-1}+1-2 \cos ^{2} \gamma}} .
\end{aligned}
$$


In order to rewrite the result in terms of the Schwarzschild radial coordinate $r$ one has to substitute here

$$
\rho=2 \frac{r^{2}}{r_{\mathrm{g}}^{2}}-1
$$

In the limit when $\rho=\rho^{\prime}$ one gets

$$
\sin \psi=\cos \gamma, \quad \varkappa=\sqrt{\frac{\rho^{2}-1}{\rho^{2}-\cos ^{2} \gamma}} .
$$

\subsection{Even $D$}

In even dimensions (odd $n$ ) the heat kernel has the form

$$
\begin{aligned}
K(s \mid \chi, \gamma)=- & 4 \frac{a^{2}}{n}\left(\frac{1}{2 \pi a^{2}} \frac{\partial}{\partial \cos \gamma}\right)^{(n+1) / 2} \frac{1}{(4 \pi s)^{2}} \sum_{k=-\infty}^{\infty}(-1)^{k} \\
& \times \int_{\chi}^{\infty} d y \frac{y e^{-a^{2} y^{2} /\left(4 n^{2} s\right)}}{(\cosh y-\cosh \chi)^{1 / 2}} \int_{\gamma}^{\pi} d \phi(\cos \gamma-\cos \phi)^{1 / 2} \frac{\partial}{\partial \phi} e^{-\frac{a^{2}(\phi+2 \pi k)^{2}}{4 s}}
\end{aligned}
$$

The Green function reads

$$
\begin{aligned}
\mathbb{G}(\chi, \gamma)=- & \frac{1}{n} \frac{1}{\pi^{2}}\left(\frac{1}{2 \pi a^{2}} \frac{\partial}{\partial \cos \gamma}\right)^{(n+1) / 2} \sum_{k=-\infty}^{\infty}(-1)^{k} \\
& \times \int_{\chi}^{\infty} d y \frac{y}{(\cosh y-\cosh \chi)^{1 / 2}} \int_{\gamma}^{\pi} d \phi(\cos \gamma-\cos \phi)^{1 / 2} \frac{\partial}{\partial \phi} \frac{1}{\left[\frac{y^{2}}{n^{2}}+(\phi+2 \pi k)^{2}\right]}
\end{aligned}
$$

The series can be computed by using the relation

$$
\sum_{k=-\infty}^{\infty} \frac{(-1)^{k}}{\left[\frac{y^{2}}{n^{2}}+(\phi+2 \pi k)^{2}\right]}=\frac{n}{2 y} \frac{\sinh \left(\frac{y}{2 n}\right) \cos \left(\frac{\phi}{2}\right)}{\cosh ^{2}\left(\frac{y}{2 n}\right)-\cos ^{2}\left(\frac{\phi}{2}\right)} .
$$

Then straightforward integration leads to the Euclidean Green function in the form

$$
\mathbb{G}(\chi, \gamma)=\frac{1}{4 \pi}\left(\frac{1}{2 \pi a^{2}} \frac{\partial}{\partial \cos \gamma}\right)^{(n+1) / 2} A_{n}
$$

where

$$
A_{n}=\int_{\chi}^{\infty} d y \frac{1}{\sqrt{\cosh ^{2}\left(\frac{y}{2}\right)-\cosh ^{2}\left(\frac{\chi}{2}\right)}} \sinh \left(\frac{y}{2 n}\right)\left[\frac{\cosh \left(\frac{y}{2 n}\right)}{\sqrt{\cosh ^{2}\left(\frac{y}{2 n}\right)-\cos ^{2}\left(\frac{\gamma}{2}\right)}}-1\right] .
$$

Because there is always at least one derivative with respect to $\gamma$ acting on $A_{n}$, for $n \geq 3$ one can safely omit the -1 in the square brackets in the definition of $A_{n}$. Therefore, for $n \geq 3$ we get

$$
A_{n}=\int_{\chi}^{\infty} d y \frac{1}{\sqrt{\cosh y-\cosh \chi}} \frac{\sinh \left(\frac{y}{n}\right)}{\sqrt{\cosh \left(\frac{y}{n}\right)-\cos \gamma}}
$$


Because the static Green functions in the Euclidean Bertotti-Robinson spacetime and in the Euclidean Tangherlini spacetime coincide, one can use the relations (4.10),(4.12) to get the static Green function on the even-dimensional Schwarzschild-Tangherlini spacetimes,

$$
G\left(x, x^{\prime}\right)=\frac{a}{2 \pi n}\left(\frac{1}{2 \pi a^{2}} \frac{\partial}{\partial \cos \gamma}\right)^{(n+1) / 2} \int_{0}^{2 \pi} d \sigma A_{n}
$$

where $x=\left(\rho, \theta_{i}\right), a=r_{\mathrm{g}}$, and

$$
\cosh (\chi)=\rho \rho^{\prime}-\sqrt{\rho^{2}-1} \sqrt{\rho^{\prime 2}-1} \cos \sigma, \quad \rho=2 \frac{r^{n}}{r_{\mathrm{g}}^{n}}-1 .
$$

\subsection{Example. $D=4$}

In four dimensions $(n=1)$ we obtain

$$
\begin{aligned}
A_{1} & =\ln \left(\frac{\cosh \chi+1}{\cosh \chi-\cos \gamma}\right), \\
\mathbb{G}(\chi, \gamma) & =\frac{1}{4 \pi}\left(\frac{1}{2 \pi a^{2}} \frac{\partial}{\partial \cos \gamma}\right) A_{1}=\frac{1}{8 \pi^{2} a^{2}} \frac{1}{\cosh \chi-\cos \gamma} .
\end{aligned}
$$

The static Green function is obtained by substitution of (5.5), and integration over $\sigma$ :

$$
\begin{aligned}
G\left(x, x^{\prime}\right) & =2 a \int_{0}^{2 \pi} d \sigma \mathbb{G}(\chi, \gamma) \\
& =\frac{1}{4 \pi^{2} a} \int_{0}^{2 \pi} d \sigma \frac{1}{\rho \rho^{\prime}-\sqrt{\rho^{2}-1} \sqrt{\rho^{\prime 2}-1} \cos \sigma-\cos \gamma} .
\end{aligned}
$$

The integral can be taken explicitly and we obtain the closed form for the static Green function

$$
G\left(x, x^{\prime}\right)=\frac{1}{2 \pi a} \frac{1}{\sqrt{\rho^{2}+\rho^{\prime 2}-2 \rho \rho^{\prime} \cos \gamma-1+\cos ^{2} \gamma}} .
$$

In four dimensions the static Green function for the Schwarzschild black hole of mass $M$ is obtained then by substitutions

$$
a=r_{\mathrm{g}}=2 M, \quad \rho=2 \frac{r}{r_{g}}-1=\frac{r}{M}-1 .
$$

In terms of the Schwarzschild coordinates it reads

$$
G\left(x, x^{\prime}\right)=\frac{1}{4 \pi} \frac{1}{\sqrt{(r-M)^{2}+\left(r^{\prime}-M\right)^{2}-2(r-M)\left(r^{\prime}-M\right) \cos \gamma-M^{2} \sin ^{2} \gamma}} .
$$

This formula exactly reproduces the closed form of the well known result for the scalar Green function in the four-dimensional Schwarzschild geometry [4, 9, 24]. 


\subsection{Example. $D=6$}

In six dimensions $(n=3)$ we obtain

$$
\begin{aligned}
A_{3} & =\int_{\chi}^{\infty} d y \frac{1}{(\cosh y-\cosh \chi)^{1 / 2}} \frac{\sinh \left(\frac{y}{3}\right)}{\sqrt{\cosh \left(\frac{y}{3}\right)-\cos (\gamma)}} \\
& =3 \int_{\cosh (\chi / 3)}^{\infty} d z \frac{1}{\sqrt{4 z^{3}-3 z-\cosh \chi}} \frac{1}{\sqrt{z-\cos \gamma}} .
\end{aligned}
$$

This integral can be expressed in terms of the elliptic function $\mathbf{F}$

$$
A_{3}=\frac{6}{\sqrt{v(w-u)}} \mathbf{F}\left(\arcsin \sqrt{\frac{w-u}{w}}, \frac{w(v-u)}{v(w-u)}\right),
$$

where

$$
p=\cosh (\chi / 3), \quad u=3 p-i \sqrt{3 p^{2}-3}, \quad v=3 p+i \sqrt{3 p^{2}-3}, \quad w=2(p-\cos \gamma) .
$$

Note that $A_{3}$ is real in spite of the complexity of the functions $u$ and $v$. The Green function on the Euclidean Bertotti-Robinson space reads

$$
\mathbb{G}(\chi, \gamma)=\frac{1}{16 \pi^{3} a^{4}}\left(\frac{\partial}{\partial \cos \gamma}\right)^{2} A_{3} .
$$

Then the static Green function in the six-dimensional Schwarzschild-Tangherlini spacetime is given by the integral

$$
G\left(x, x^{\prime}\right)=\frac{1}{24 \pi^{3} a^{3}}\left(\frac{\partial}{\partial \cos \gamma}\right)^{2} \int_{0}^{2 \pi} d \sigma A_{3},
$$

where $a=r_{\mathrm{g}}$.

It is problematic to obtain an answer for the Green functions in a closed form for $D \geq 6$. However a simple integral representation is possible in all dimensions what may be good enough for some applications like computing of the self-force and self-energy of scalar charges in the Schwarzschild-Tangherlini spacetime.

\section{Discussion}

In this paper we discuss properties of a field created by a static source placed in the vicinity of a static higher-dimensional black hole. We focused on a static Green function which allows one to find the field of a point charge. If the spacetime has $D$ dimensions, such a $(D-1)$-dimensional static Green function can be obtained by the dimensional reduction from a Green function in $D$ dimensions. We demonstrated that there exists a group of transformations of the original $D$ dimensional metric, depending on a function of $(D-1)$ variables, which preserves the form of a static $(D-1)$ dimensional field equation. This transformation consists of a conformal transformation of the spatial part of the metric accompanied by a properly chosen transformation of a red-shift factor (i.e. $g_{t t}$ component 
of the metric). These transformations change the $D$ dimensional metric. In a general case, after such a transformation, a solution of the Einstein equations is transformed to a metric that does not satisfy the Einstein equations with any physically meaningful stress-energy tensor. We call these transformations bi-conformal. We demonstrated that the metrics describing higher-dimensional static black holes (Schwarzschild-Tangherlini metrics and their electrically charged generalizations) possess a remarkable property: within a family of metrics related to such black hole metrics by means of a bi-conformal transformation there exist special metrics that have enhanced symmetry. Namely, the symmetry $\operatorname{SO}(D-1) \times R^{1}$ of the original spacetime is enhanced up to $\mathrm{SO}(D-1) \times \mathrm{SO}(1,2)$. The spacetime with this symmetry is a Bertotti-Robinson metric that is a direct sum of the metrics on a $(D-2)$ dimensional sphere and 2-dimensional AdS spacetime. We used the heat kernel method to obtain a useful representation for the Green function in the higher-dimensional BertottiRobinson spacetime and after its dimensional reduction we found the static Green function in the original black hole metric. In four and five dimensions we obtained a closed form for the static Green functions in terms of elementary and elliptic functions respectively.

The described method is quite general. We illustrated its application by considering a minimally coupled scalar massless field from a static point charge located in the vicinity of the higher-dimensional Schwarzschild-Tangherlini black hole. Generalizations to charged black holes and to the case of an electric point charge, which are rather straightforward, will be discussed elsewhere. Let us also mention that if one applies the static Green function for the calculation of the self-energy and self-force for a point charge, one should first regularize it and subtract local divergences. This procedure depends on the choice of the background $D$-dimensional metric. As a result, renormalized self-energy and self-force in two spacetimes connected by a bi-conformal transformation are not connected by simple rescaling. This phenomenon, which is similar to well known conformal anomalies, is called a bi-conformal anomaly or self-energy anomaly (see discussion in [15-17]). We are going to return to this problem again in future works.

\section{Acknowledgments}

This work was partly supported by the Natural Sciences and Engineering Research Council of Canada. The authors are also grateful to the Killam Trust for its financial support. The authors thank Don Page for useful comments on the paper.

\section{A Useful properties of the elliptic functions}

Calculating the static Green function (6.13) in the section 6.2 we have used the integrals

$$
\begin{aligned}
\int_{0}^{\pi / 2} d x \frac{1}{\sqrt{1-k^{2} \sin ^{2} x}} \arctan \left(\frac{1}{\alpha \sqrt{1-k^{2} \sin ^{2} x}}\right) & =\frac{\pi}{2} \mathbf{F}\left(\arctan \left(\frac{1}{\alpha \sqrt{1-k^{2}}}\right), k\right), \\
\int_{0}^{\pi / 2} d x \frac{1}{\sqrt{1-k^{2} \sin ^{2} x}} & =\mathbf{F}\left(\frac{\pi}{2}, k\right)=\mathbf{K}(k)
\end{aligned}
$$


The Green function (6.13) takes the form

$$
G\left(x, x^{\prime}\right)=\frac{1}{4 \pi^{2} a^{2}}\left(\frac{\partial}{\partial \cos \gamma}\right)\left\{\frac{1}{\alpha \cos \gamma}[\mathbf{F}(\phi, k)+\mathbf{K}(k)]\right\},
$$

where

$$
\begin{aligned}
\phi & =\arctan \left(\frac{1}{\alpha \sqrt{1-k^{2}}}\right), \\
\alpha^{2} & =\frac{1}{2 \cos ^{2} \gamma}\left(\rho \rho^{\prime}-\sqrt{\rho^{2}-1} \sqrt{\rho^{\prime 2}-1}+1-2 \cos ^{2} \gamma\right), \\
k^{2} & =-\frac{2 \sqrt{\rho^{2}-1} \sqrt{\rho^{\prime 2}-1}}{\rho \rho^{\prime}-\sqrt{\rho^{2}-1} \sqrt{\rho^{\prime 2}-1}+1-2 \cos ^{2} \gamma} .
\end{aligned}
$$

One gets

$$
\sin \phi=\frac{1}{\sqrt{1+\alpha^{2}\left(1-k^{2}\right)}}=\cos \gamma \frac{\sqrt{2}}{\sqrt{\rho \rho^{\prime}+\sqrt{\rho^{2}-1} \sqrt{\rho^{\prime 2}-1}+1}} .
$$

It is convenient to use the identity

$$
\mathbf{F}(\phi, k)=\frac{1}{k^{\prime}} \mathbf{F}\left(\psi, i \frac{k}{k^{\prime}}\right)
$$

with

$$
k^{\prime}=\sqrt{1-k^{2}}, \quad \sin \psi=k^{\prime} \frac{\sin \phi}{\sqrt{1-k^{2} \sin ^{2} \phi}},
$$

or, equivalently,

$$
\begin{aligned}
k^{\prime 2} & =\frac{\rho \rho^{\prime}+\sqrt{\rho^{2}-1} \sqrt{\rho^{\prime 2}-1}+1-2 \cos ^{2} \gamma}{\rho \rho^{\prime}-\sqrt{\rho^{2}-1} \sqrt{\rho^{\prime 2}-1}+1-2 \cos ^{2} \gamma} \\
\sin \psi & =\frac{1}{\sqrt{1+\alpha^{2}}}=\cos \gamma \frac{\sqrt{2}}{\sqrt{\rho \rho^{\prime}-\sqrt{\rho^{2}-1} \sqrt{\rho^{\prime 2}-1}+1}} .
\end{aligned}
$$

One can rewrite the static Green function as

$$
G\left(x, x^{\prime}\right)=\frac{1}{4 \pi^{2} a^{2}}\left(\frac{\partial}{\partial \cos \gamma}\right)\left\{\frac{\sqrt{2}}{\sqrt{\rho \rho^{\prime}+\sqrt{\rho^{2}-1} \sqrt{\rho^{\prime 2}-1}+1-2 \cos ^{2} \gamma}}[\mathbf{F}(\psi, \varkappa)+\mathbf{K}(\varkappa)]\right\} .
$$

Here

$$
\varkappa=i \frac{k}{k^{\prime}}=\sqrt{\frac{2 \sqrt{\rho^{2}-1} \sqrt{\rho^{\prime 2}-1}}{\rho \rho^{\prime}+\sqrt{\rho^{2}-1} \sqrt{\rho^{\prime 2}-1}+1-2 \cos ^{2} \gamma}},
$$

Eventually we obtain the static Green function

$$
G\left(x, x^{\prime}\right)=\frac{1}{4 \pi^{2} a^{2}} \frac{1}{\left(\rho^{2}-1\right)^{1 / 4}\left(\rho^{\prime 2}-1\right)^{1 / 4}}\left(\frac{\partial}{\partial \cos \gamma}\right)\{\varkappa[\mathbf{F}(\psi, \varkappa)+\mathbf{K}(\varkappa)]\} .
$$

In this representation $0 \leq \varkappa \leq 1$. 
Open Access. This article is distributed under the terms of the Creative Commons Attribution License (CC-BY 4.0), which permits any use, distribution and reproduction in any medium, provided the original author(s) and source are credited.

\section{References}

[1] W.G. Unruh, Selfforce on charged particles, Proc. Roy. Soc. Lond. A 348 (1976) 447 [INSPIRE].

[2] A.G. Smith and C.M. Will, Force on a static charge outside a Schwarzschild black hole, Phys. Rev. D 22 (1980) 1276 [INSPIRE].

[3] A. Zelnikov and V. P. Frolov, The influence of gravitation, acceleration, and temperature on the self-energy of charged particles (in Russian), Proc. Lebedev Phys. Inst. 152 (1983) 96.

[4] A. Zelnikov and V. Frolov, Influence of gravitation on the self-energy of charged particles, Sov. Phys. JETP 55 (1982) 191.

[5] E.T. Copson, On electrostatics in a gravitational field, Proc. Roy. Soc. London A 118 (1928) 184.

[6] M.J.S. Beach, E. Poisson and B.G. Nickel, Self-force on a charge outside a five-dimensional black hole, Phys. Rev. D 89 (2014) 124014 [arXiv:1404.1031] [INSPIRE].

[7] V. Frolov and A. Zelnikov, Charged particles in higher dimensional homogeneous gravitational field: self-energy and self-force, JHEP 1410 (2014) 68 [arXiv:1407.3323] [INSPIRE].

[8] B. Linet, Electrostatics and magnetostatics in the Schwarzschild metric, J. Phys. A 9 (1976) 1081 [INSPIRE].

[9] B. Linet, Scalar or electric charge at rest in a black hole space-time, Compt. Rend. Math. 284 (1977) 215.

[10] A.C. Ottewill and P. Taylor, Static Kerr Green's function in closed form and an analytic derivation of the self-force for a static scalar charge in Kerr space-time, Phys. Rev. D 86 (2012) 024036 [arXiv: 1205.5587] [INSPIRE].

[11] V.P. Frolov and A. Zelnikov, Scalar and electromagnetic fields of static sources in higher dimensional Majumdar-Papapetrou spacetimes, Phys. Rev. D 85 (2012) 064032 [arXiv: 1202.0250] [INSPIRE].

[12] A. Garcia-Parrado and J.M.M. Senovilla, Bi-conformal vector fields and their applications, Class. Quant. Grav. 21 (2004) 2153 [math-ph/0311014] [INSPIRE].

[13] A.G.P. Gomez-Lobo, Bi-conformal vector fields and the local geometric characterization of conformally separable pseudo-riemannian manifolds I, J. Geom. Phys. 56 (2006) 1069.

[14] V.P. Frolov and A. Zelnikov, Self-energy of a scalar charge near higher-dimensional black holes, Phys. Rev. D 85 (2012) 124042 [arXiv:1204.3122] [InSPIRE].

[15] V.P. Frolov and A. Zelnikov, Classical self-energy and anomaly, Phys. Rev. D 86 (2012) 044022 [arXiv: 1205.4269] [INSPIRE].

[16] V.P. Frolov and A. Zelnikov, Anomaly and the self-energy of electric charges, Phys. Rev. D 86 (2012) 104021 [arXiv:1208.5763] [INSPIRE]. 
[17] V.P. Frolov, A.A. Shoom and A. Zelnikov, Self-energy anomaly of an electric pointlike dipole in three-dimensional static spacetimes, Phys. Rev. D 88 (2013) 024032 [arXiv:1303.1816] [INSPIRE].

[18] M. Demianski and I. Novikov, Electric charge in the kruskal space-time and the jeans conjecture, Gen. Rel. Grav. 14 (1982) 1115.

[19] V. Frolov and I. Novikov, Black hole physics: basic concepts and new developments, Fundamental Theories of Physics vol. 96, Kluwer Academic Publishers, Dordrecht Netherlands (1998).

[20] E. Poisson, A. Pound and I. Vega, The motion of point particles in curved spacetime, Living Rev. Rel. 14 (2011) 7 [arXiv:1102.0529] [INSPIRE].

[21] R. Camporesi, Harmonic analysis and propagators on homogeneous spaces, Phys. Rept. 196 (1990) 1 [INSPIRE].

[22] L.A. Kofman and V. Sahni, A new selfconsistent solution of the Einstein equations with one loop quantum gravitational corrections, Phys. Lett. B 127 (1983) 197 [INSPIRE].

[23] A.C. Ottewill and P. Taylor, Quantum field theory on the Bertotti-Robinson space-time, Phys. Rev. D 86 (2012) 104067 [arXiv:1209.6080] [INSPIRE].

[24] V.P. Frolov and A.I. Zel'nikov, The massless scalar field around a static black hole, J. Phys. A 13 (1980) L345. 